\begin{tabular}{|c|c|}
\hline Title & $\begin{array}{l}\text { In vitro biomechanical effects of reconstruction on adjacent motion segment: comparison of aligned/kyphotic } \\
\text { posterolateral fusion with aligned posterior lumbar interbody fusion/posterolateral fusion. }\end{array}$ \\
\hline Author(s) & Sudo, Hideki; Oda, I.; A bumi, K.; Ito, M. \\
\hline Citation & $\begin{array}{l}99 \text { (2 suppl.), } 221-28 \\
\text { https://doi.org/10.3171/spi.2003.99.2.0221 }\end{array}$ \\
\hline Issue Date & $2003-09$ \\
\hline Doc URL & http:/hdl.handle.net/2115/44599 \\
\hline Rights & $\begin{array}{l}\text { The final version of the paper was published in (Journal of Neurosurgery, vol.99-2). For reuse of any of the materials, } \\
\text { including editorial copy, figures, or tables please contact the Journal of Neurosurgery at jneuro@virginia.edu }\end{array}$ \\
\hline Type & article (author version) \\
\hline File Information & JN2003-99-2.pdf \\
\hline
\end{tabular}

Instructions for use 


\title{
In vitro biomechanical effects of reconstruction on adjacent motion segment: comparison of aligned/ kyphotic posterolateral fusion with aligned posterior lumbar interbody fusion/ posterolateral fusion
}

\author{
Hideki Sudo, M.D., I taru Oda, M.D., Kuniyoshi Abumi, M.D., Manabu Ito, \\ M.D., Yoshihisa Kotani, M.D., Yoshihiro Hojo, M.D. and Akio Minami, \\ M.D. \\ Department of Orthopaedic Surgery, Hokkaido University Graduate \\ School of Medicine, Sapporo, Japan
}

OBJECT. Posterior lumbar interbody fusion (PLIF) was developed to overcome the limitations of posterolateral fusion in correcting spinal deformity and maintaining lumbar lordosis. In this study the authors compare the biomechanical effects of three different posterior reconstructions on the adjacent motion segment.

METHODS. Ten calf spinal (L2-S1) specimens underwent nondestructive flexion-extension testing ( $\pm 6 \mathrm{Nm}$ ). The specimens were destabilized at the L5-S1 levels after intact testing. This was followed by pedicle screw fixation with and without interbody cages as follows: 1) with straight rods ("aligned" posterolateral fusion); 2) with kyphotically prebent rods ("kyphotic" posterolateral fusion); and 3) with interbody cages combined with straight rods ("aligned" PLIF/posterolateral fusion). The range of motion (ROM) of the operative segments, the intradiscal pressure (IDP), and longitudinal lamina strain in the superior adjacent segment (L4-5) were analyzed.

The ROM associated with aligned PLIF/posterolateral fusion-treated specimens was significantly less than both the aligned and kyphotic posterolateral fusion-treated procedures in both flexion and extension loading ( $p \leqslant 0.05$ ). The aligned PLIF/posterolateral fusion was associated with greater IDP and the lamina strain compared with the aligned and kyphotic posterolateral fusion groups in flexion loading. Under extension loading, greater IDP and lamina strain were present in the kyphotic posterolateral fusion group than in the aligned posterolateral fusion group. The highest IDP and lamina strain were shown in the aligned PLIF/posterolateral fusion group. 
CONCLUSIONS. Compared with kyphotic posterolateral fusion, PLIF may lead to even higher load at the superior adjacent level because of the increased stiffness of the fixed segments even if local kyphosis is corrected by PLIF.

\section{KEY WORDS. posterolateral fusion, posterior lumbar interbody fusion, kyphosis, biomechanics, intradiscal pressure \\ ABBREVI ATI ONS. ALL = anterior longitudinal ligament, I DP = intradiscal pressure, PLIF = posterior lumbar interbody fusion, ROM = range of motion, SD = standard deviation}

Posterolateral fusion involving the pedicle screw system has been widely used in the treatment of spinal instability caused by degenerative disorders, tumors, and traumatic injury. It has been reported that pseudarthrosis, a progressive loss of correction, and instrumentation failure, however, may occur in 7 to $10 \%$ of cases. $\underline{21}, \underline{33}$

Several authors have reported that PLIF combined with posterolateral fusion (PLIF/posterolateral fusion) provides anterior structural support, maintains the lumbar lordosis,,$\frac{30}{}$ increases construct-related stiffness, $\underline{2}$ and improves fusion rate ${ }^{30}$ compared with posterolateral fusion alone. It is widely believed, however, that spinal fusion increases biomechanical stress on the adjacent unfused motion segments and causes early degenerative discal and facet joint changes. $\underline{7} \underline{10}, \underline{18}, \underline{20}$ Furthermore, considerably high PLIF-related complication rates have been reported, including nerve root injury due to cage insertion and migration of the cages into the spinal canal. ${ }^{9}, \underline{25}$ It has thus been suggested that the spinal reconstruction technique should be selected on a case-by-case basis.

Several in vitro studies have been conducted to investigate the effects of interbody fusion on the adjacent segments. Chow, et al., ${ }^{4}$ measured the segmental mobility and IDP in cadaveric lumbar spine following anterior interbody fusion, and demonstrated an increased load on the neighboring unfused segments. Only anterior interbody fusion without posterior pedicle screw instrumentations, however, was simulated in their study. Few studies to date have been undertaken to compare the effects of posterolateral fusion and PLIF/posterolateral fusion on the adjacent segments. 
It is accepted that spinal fusion does not always produce good results. Sagittal-plane imbalance is a major problem. $\underline{15-17}, \underline{27}, \underline{28}, \underline{34}$ In a clinical follow-up study, Kumar, et al., $\underline{16}$ examined the relationship between changes in sagittal-plane configuration of the lumbar spine and the development of adjacent-segment degeneration following posterolateral fusion with or without PLIF. They concluded that normality of sacral inclination is an important parameter for minimizing degeneration. In their study, however, the specific configuration of the fused segments was not investigated. Therefore, it remains uncertain whether local malalignment of the fused segments after lumbar spinal fusion promotes a higher incidence of early adjacent-segment degeneration.

No biomechanical study has been conducted to compare the effects of residual local kyphosis after posterolateral fusion and physiological alignment restored by PLIF on the adjacent segments.

The purposes of present study were to compare three different posterior lumbar reconstruction procedures--aligned posterolateral fusion, kyphotic posterolateral fusion, and aligned PLIF/posterolateral fusion--to ascertain their biomechanical effects on the adjacent motion segment and to discuss indications for posterolateral fusion and PLIF procedures.

Materials and Methods

\section{Specimen Preparation and Biomechanical Testing}

Ten fresh calf spines (L2-S1 segments obtained in animals 8-10 weeks of age at the time of death) were stored frozen in double-wrapped plastic bags at $-25^{\circ} \mathrm{C}$. Prior to mechanical testing, specimens were thawed to room temperature, and the soft tissues were removed, taking care not to damage the disc, ligaments, or joint capsules. They were kept moist by using a saline atomizer throughout testing. Each specimen was embedded in polyester resin (Flexible Body Filler of Polyester Resin; Soler Co., Ltd., Tokyo, Japan) superior to L-2 and inferior to S-1 and securely fastened onto a testing table (Fig. 1 left). Radiographs were obtained in each case to ensure that the L4-5 intervertebral disc was oriented horizontally. Nondestructive 
biomechanical testing was performed by applying two types of pure moments onto the multisegmental (L2-S1) specimens in a manner previously reported. $\underline{1}, \underline{23}, \underline{26}$ The loading modes included flexion $(6 \mathrm{Nm})$ and extension $(6 \mathrm{Nm})$. The maximum loading levels were within the range of those reported in previous biomechanical studies involving lumbar reconstruction models. 1 , 11, 13, 14, 23 Because pilot studies demonstrated that load-displacement and load-strain or pressure data after the second load-unload cycle were almost identical, the loading procedure involved the application of three load- unload cycles. The third loading cycle was used to allow for data analysis of all biomechanical parameters. The specimen was allowed to "creep" for 30 seconds between load application and data collection. $\underline{13}, \underline{14}, \underline{23}$

Two-level fusion was simulated at L5-6 and L6-S1. The L4-5 level was defined as the superior adjacent segment. Uniaxial surface-strain gauges

(KFG-02-120-C1-11L3M2R; Kyowa Dengyo Co., Tokyo, Japan) were sagittally mounted on the bilateral L-4 lamina just superior to the L4-5 facet joint by using cyanoacrylate in a manner previously described. ${ }^{3}, \underline{24}$ The longitudinal lamina strains at L-4 were recorded during flexion-extension testing, which indirectly indicate load transmission through the posterior column at the adjacent segment $\underline{24}$, $\underline{31}$ (Fig. 1 center).

A specially designed pressure needle transducer (R. Denton, Inc., Rochester Hills, MI) was used to obtain the pressure within the intervertebral disc. The needle was $2.1 \mathrm{~mm}$ in diameter. Its tip housed a waterproof-coated single-strain gauge containing a 1.5- $\mathrm{mm}$-diameter sensing area. A clear epoxy resin was used to mount the transducer. The needle was initially calibrated to $1.36 \mathrm{MPa}$ with a hydraulic test. Details of the needle have been described previously. ${ }^{7}$ The pressure needle was inserted from the lateral side of the L4-5 disc into the center of the nucleus pulposus in a manner previously described ${ }^{\underline{7}}$ (Fig. 1 right). The insertion point and depth of the needle were controlled using both anteroposterior and lateral radiographs.

Segmental displacement at L5-S1 was also measured using an anteriorly positioned displacement gauge (Model 25C-20; MTS Systems, Inc., Minneapolis, MN) bridging the L- 5 and S- 1 vertebral bodies through the anterior longitudinal ligament. The ROM was measured in relation to the corresponding anterior longitudinal ligament displacement (millimeters) to evaluate the stability of the fixed segments. 
Data were acquired through surface-strain gauges and the pressure needle transducer connected to a multichannel signal-conditioning sensor board (PCD-200A; Kyowa Dengyo Co., Tokyo, Japan) interfaced with a personal computer (PC-MJ 710R; Sharp Inc., Tokyo, Japan), and recorded simultaneously with segmental displacement data. For the lamina strain analysis, the mean values of right and left gauges were calculated to minimize the effects of coupled motions.

\section{Experimental Stages}

After the intact specimen was tested, destabilization was performed. Transection of supra- and interspinous ligaments, following bilateral partial facetectomy and discectomy, was performed at L5-6 and L6-S1. The anterior outer layer of the anulus fibrosus and the ALL were preserved. These conditions simulated both posterior- and anterior-column instability. After the discectomy, three types of spinal reconstruction were sequentially performed on each specimen. The order of this sequence was randomized among specimens. The fixation procedure involved placement of a pedicle screw fixation system and interbody fusion cages as follows: 1) L5-S1 aligned pedicle screw fixation (aligned posterolateral fusion); 2) L5-S1 pedicle screw fixation with kyphosis (kyphotic posterolateral fusion); and 3) L5-S1 aligned pedicle screw fixation with interbody fusion cages (aligned PLIF/posterolateral fusion) (Fig. 2).

The posterolateral fusion was simulated by pedicle screw fixation, and the PLIF by placement of interbody fusion cages. To perform the fixation procedure, titanium pedicle screws (Isola; Depuy-AcroMed, Boston, MA [6.25 mm in diameter, $45 \mathrm{~mm}$ in length]) were inserted bilaterally at L-5, L-6, and S-1 parallel to the endplate. The screws inserted at L-5 did not damage the L4-5 facet joint capsule. For the aligned posterolateral fusion, straight titanium rods (I sola; Depuy-AcroMed [6.35 mm in diameter]) were used. For the kyphotic posterolateral fusion, kyphotically prebent rods were applied to target $30^{\circ}$ at the operative segments, as previously described. $\frac{24}{4}$ In cases of aligned PLIF/posterolateral fusion, two carbon fiber rectangular cages (Brantigan cage; Depuy-AcroMed [top surface dimension $9 \times 21 \mathrm{~mm}$; height $10 \mathrm{~mm}$ ]) were inserted into each disc bilaterally by following the manufacturer's instructions; straight titanium rods were used. Using the Bionix 858 biaxial servohydrolic materials test machine (MTS), a $125-\mathrm{N}$ axial compression force was applied to the specimen when tightening the rod-screw junctions. This preloading level value was chosen after referring to a previous biomechanical study involving intervertebral cages. $\frac{5}{5}$ 
Biomechanical testing was repeated after each reconstruction and permitted each specimen to serve as its own control, ensuring data normalization.

\section{Statistical Analysis}

Statistical significance was determined using a repeated-measures analysis of variance in addition to a post hoc multiple comparison involving the Fisher protected least significant difference test with a 95\% confidence level.

\section{Results}

\section{Angle at the Operative Segments}

The actual sagittal angle at the surgical site in each specimen was measured on plain lateral radiographs by using the Cobb angle at L5-S1. The mean angles ( \pm SD) were $0.4 \pm 0.84^{\circ}$ of lordosis in the intact group, $2.2 \pm 0.63^{\circ}$ of lordosis in the aligned posterolateral fusion group, $28.3 \pm 0.95^{\circ}$ of kyphosis in the kyphotic posterolateral fusion group, and $2.8 \pm 0.79^{\circ}$ of lordosis in the aligned PLIF/ posterolateral fusion group.

\section{Operative Segment ROM}

The ROMs of the operative segments varied significantly among the four groups under flexion loading ( $F=26.770, p<0.0001)$. The aligned posterolateral fusion, kyphotic posterolateral fusion, and aligned PLIF/posterolateral fusion models exhibited statistically less ROM than the intact condition before treatment ( $p<0.05$ ). The ROM of the aligned PLIF/posterolateral fusion was significantly less than that of either aligned posterolateral fusion or kyphotic posterolateral fusion ( $p<0.05$ ). No statistical significance was demonstrated between mean values in the aligned posterolateral fusion and kyphotic posterolateral fusion models (Fig. 3 upper). 
Similar to the flexion loading values, the ROM of the operative segments varied significantly among the four groups under extension loading ( $F=10.835, p \leqslant 0.0001)$. Statistically less ROM was demonstrated in the aligned PLIF/posterolateral fusion models than in the intact, aligned posterolateral fusion, and kyphotic posterolateral fusion models ( $p<0.05$ ). No statistical significance was detected between the intact, aligned posterolateral fusion, and kyphotic posterolateral fusion models (Fig. 3 lower).

\section{Intradiscal Pressure}

An analysis of IDP within the adjacent segment demonstrated significant differences among the four groups under flexion loading $(F=7.529, p=0.0005)$. All reconstruction models showed significantly higher IDP within the superior adjacent segment than in the intact condition $(p * 0.05)$. The adjacent IDP of the aligned PLIF/posterolateral fusion model was significantly higher than that in the intact, aligned posterolateral fusion, or kyphotic posterolateral fusion models ( $p \leqslant 0.05$ ). No statistical difference was observed between the aligned posterolateral fusion and kyphotic posterolateral fusion models (Fig. 4 upper).

Extension loading also showed significant differences among the four treatment groups ( $F=3.591, p=0.0228$ ). A significant increase in the IDP was found in the kyphotic posterolateral fusion and aligned PLIF/posterolateral fusion models compared with the intact condition ( $p<0.05$ ). The aligned posterolateral fusion model showed no significantly higher IDP compared with that in the intact condition. The IDPs demonstrated in the kyphotic posterolateral fusion and aligned PLIF/posterolateral fusion models were higher than that in the aligned posterolateral fusion model; however, statistical significance was not reached (Fig. 4 lower).

\section{Lamina Strain}

Under flexion loading, testing of the tensile lamina strain at the adjacent segment demonstrated statistical differences among the four groups $(F=3.918, p=0.0161)$. Although all reconstruction models increased the lamina strain under flexion loading, only the aligned PLIF/posterolateral fusion model was associated with significantly higher strain compared with the intact state $(p<0.05)$. No difference was detected 
between the aligned posterolateral fusion, kyphotic posterolateral fusion, and aligned PLIF/posterolateral fusion models (Fig. 5 upper).

In addition, extension load testing demonstrated significant differences among the four groups $(F=3.928, p=0.0159)$. A significant increase in compression strain of the lamina was found in the kyphotic posterolateral fusion and aligned PLIF/posterolateral fusion models compared with the intact specimens ( $p<0.05$ ). No significantly higher lamina strain was observed in the aligned posterolateral fusion model than in the intact specimens. There was no significant difference among the aligned posterolateral fusion, kyphotic posterolateral fusion, and aligned PLIF/posterolateral fusion models (Fig. 5 lower).

Discussion

\section{Study Design}

The authors of many biomechanical studies have reported that spinal instrumentation leads to higher construct stiffness, which leads to hypermobility of the adjacent unfused segments. ${ }^{4}, \underline{8}, \underline{19}, \underline{22}, \underline{29}$ It has also been documented that spinal malalignment after the initial spinal instrumentation may accelerate adjacent-segment degenerative changes. ${ }^{24}, \underline{31}$ Using an in vivo sheep model, Oda, et al., ${ }^{24}$ reported that spinal kyphotic deformity led to an increased load transmission via the posterior column and resulted in lordotic contracture of the posterior ligamentous complex at the superior adjacent motion segment. These findings have suggested that both construct stiffness and physiological alignment of the fused segments are important factors affecting the degenerative changes in the adjacent segments.

By combining a PLIF procedure with the conventional posterolateral fusion, one may add the risk of promoting degenerative changes in the adjacent unfused motion segments because of the increased stress caused by the stiffness of the fused segments. There have been few studies, however, in which investigators compared posterolateral fusion and PLIF in terms of their effects on the adjacent motion 
segments. Additionally, no study has, to date, been undertaken to compare the effects of residual kyphosis on the adjacent segments after posterolateral fusion and Iordosis restored by PLIF. In the present study, spinal destabilization was performed and followed by pedicle screw fixation both with and without interbody cages. Aligned posterolateral fusion was simulated using pedicle screws and straight rods. Residual local kyphosis following posterolateral fusion was simulated using kyphotically prebent rods. The correction of local kyphosis was simulated using straight rods and interbody cages. The effects of spinal reconstructions on the adjacent segment were then compared.

In this study, IDP and lamina strain were investigated by measuring these biomechanical parameters in the adjacent unfused superior segments and indirectly evaluating the load transmission through both anterior and posterior columns. In several in vitro studies investigators have measured IDP adjacent to the spinal fusion site. ${ }^{4}, \underline{7}, \underline{32}$ Cunningham, et al., $\underline{7}$ reported the effects of spinal destabilization and instrumentation on the adjacent IDP in human cadaveric lumbar spines. Compared with the intact spine, a significantly higher pressure was observed with hook or pedicle screw instrumentation in the axial compression and anterior flexion loading. These reports suggested that IDP was a sensitive parameter indicating changes in load transmission through adjacent segments.

\section{Biomechanical Analysis}

During flexion-extension loading, no statistical differences were found in ROM between aligned and kyphotic posterolateral fusion groups. In the kyphotic posterolateral fusion model, however, higher IDP and lamina strain were observed compared with the aligned posterolateral fusion model when extension loading was performed. Using a human cadaveric model, Umehara, et al., ${ }^{31}$ also reported that hypolordosis in the instrumentation-treated segments increased the load across the posterior transpedicular devices and increased lamina strain in the adjacent segment. These results suggest that residual kyphosis of the operative segments after posterolateral fusion alone causes excessive load on the superior adjacent segment.

The ROM values determined in the aligned PLIF/posterolateral fusion-treated region was significantly smaller than that in the aligned posterolateral fusion model under flexion loading. This finding suggests that the aligned PLIF/posterolateral fusion 
models provided significantly greater stiffness than aligned posterolateral fusion procedure in flexion loading. Using an in vitro calf lumbar spine model, Brodke, et al., $\underline{2}$ demonstrated that one-level pedicle screw fixation combined with posterior interbody cages provided 1.35 -fold greater stiffness than pedicle screw fixation alone in flexion-extension loading. These findings suggest that the intervertebral cages significantly increased the stiffness of the instrumented segments. In the present study, we found that stiffness in the aligned PLIF/ posterolateral fusion groups was 2.79- to 3.7-fold greater than that in the aligned posterolateral fusion groups under flexion-extension loading. The reason for the difference between results presented here and by Brodke, et al., may be related to the mechanical instability of the anterior column. In the model studied by Brodke, et al., destabilization of the posterior column was performed; however, the anterior column remained well preserved. Therefore, construct stiffness in our aligned posterolateral fusion model may have been less than that in the posterolateral fusion model investigated by Brodke, et al., and may explain the disparity between the posterolateral fusion and the PLIF/ posterolateral fusion models. Furthermore, we performed two-level fusion, whereas Brodke, et al., performed a one-level fusion only. This may also contribute to the differences, because the facet load on the proximal segments becomes greater as the number of immobilized segments increases. $\underline{22}$

The IDP was significantly higher in the aligned PLIF/ posterolateral fusion group than that in the aligned posterolateral fusion group in flexion loading. There was no significant difference, however, between the two reconstruction techniques when tested in extension loading. These results may arise from the effect that the cages mainly increased flexion-related stiffness of the instrumented segments.

Specimens in the aligned PLIF/posterolateral fusion group showed a statistically higher IDP in the adjacent segment than those in the kyphotic posterolateral fusion group in flexion loading. Furthermore, in specimens in the aligned PLI F/posterolateral fusion group the highest tensile lamina strain was exhibited among all groups. These results suggest that additional PLIF may lead to higher adjacent-level load transmission via both anterior and posterior columns due to the increased stiffness of the fixed segments, even if the local kyphosis is corrected by PLIF.

\section{Clinical Relevance}


The authors of some clinical studies have documented that both a high degree of construct stiffness after interbody fusion and abnormal sagittal alignment are the underlying causes for the adjacent-segment degeneration. $\underline{15-17}, \underline{27}, \underline{28}, \underline{34}$ In a retrospective study, Rahm and Hall $\underline{27}$ reported that addition of PLIF to conventional posterolateral fusion was associated with increased degeneration compared with posterolateral fusion alone. Also in a retrospective study, Kawakami, et al., $\frac{15}{}$ found a correlation between the lumbar lordosis at the fused segments and clinical outcome after posterolateral fusion for degenerative lumbar spondylolisthesis. The authors suggested that it is useful to produce postoperative physiological alignment of the fused segment. In the present study, we demonstrated that both kyphotic posterolateral fusion and aligned PLIF/posterolateral fusion can produce greater biomechanical effects on the adjacent segment compared with aligned posterolateral fusion alone and that these findings are consistent with previous clinical investigations. It remains unclear, however, whether the residual kyphosis following posterolateral fusion should be restored by performing PLIF. There have been no data regarding this issue in the literature. Our findings indicate that the PLIF/posterolateral fusion provided a high level of initial stability and corrected local kyphosis; however, additional interbody fusion might result in earlier, more severe degeneration of the adjacent segments than posterolateral fusion alone. To avoid additional surgery, detailed surgical planning should be conducted, especially if degenerative changes in the adjacent segment can already be observed.

\section{Study Limitations}

Although human cadaveric specimens are anatomically ideal for biomechanical study, a calf spine model has previously been shown to be an appropriate alternative for biomechanical testing. $\frac{12}{~ T h e ~ c a l f ~ s p i n e ~ m o d e l ~ o f f e r s ~ m a n y ~ a d v a n t a g e s ~ i n c l u d i n g ~}$ greater availability, greater specimen-to-specimen continuity, and an absence of degenerative and pathological lesions.

We measured the segmental displacement with only a single anterior displacement gauge. If a treatment alters the location of the axis of rotation, the anterior displacement could increase or decrease despite the same amount of angular spinal displacement. The single gauge is not able to measure the location of the axis of rotation, which could be changed in various spinal reconstructions. A second 
displacement gauge on the posterior elements or a three-dimensional tracking devise will be needed for more accurate measurement.

In the present study, only flexion-extension loading was performed because alignment of the fixed segments was changed on the sagittal plane. The effects under lateral bending or axial rotation remain unclear.

An in vitro cadaveric model was used and the mechanical effects of the spinal fixation were evaluated, which simulated immediate postoperative conditions. Therefore, the long-term biomechanical effects of spinal fusion on the adjacent segments remain unclear, because the effect of osseous fusion could not be investigated. In an in vivo study conducted by Cunningham, et al., $\frac{6}{a}$ higher fusion rate was accompanied by statistically increased segmental stiffness when an interbody autograft-packed fusion cage was compared with the cage alone. In the present study we failed to show a statistical difference between the kyphotic posterolateral fusion and the aligned PLIF/posterolateral fusion in the compressive lamina strain under extension loading. The construct stiffness of the aligned PLIF/posterolateral fusion under extension, however, might be expected to be higher when solid interbody fusion is achieved and may result in a higher lamina strain in the adjacent segment than in the kyphotic posterolateral fusion.

The effects of alignment and stiffness of the fused segments on the adjacent level segment were analyzed in terms of IDP and longitudinal lamina strain. Anterior and posterior shear forces on the adjacent segment, however, were not investigated. In the upright standing position, the anterior shear force would be increased if the alignment of the fused segments is kyphotic, and it may lead to more severe degenerative spondylolisthesis. Therefore, PLIF-induced correction of the local kyphosis may well have advantages over posterolateral fusion with residual kyphosis. Further biomechanical investigations are needed to clarify these points. 
Several findings were made in the present study. 1) The kyphotic posterolateral fusion was associated with a higher IDP and lamina strain compared with the aligned posterolateral fusion. 2) The aligned PLIF / posterolateral fusion was associated with a higher IDP and lamina strain compared with that in the aligned posterolateral fusion. 3) The aligned PLIF/posterolateral fusion model demonstrated an even greater IDP and lamina strain in the adjacent segment compared with the kyphotic posterolateral fusion. 4) Additional PLIF may lead to higher levels of adjacent-segment load transmission through both anterior and posterior columns due to an increase in stiffness of the fixed segments, even if the local kyphosis is corrected by PLIF. To avoid additional surgery, detailed surgical planning should be performed, especially if any degenerative changes in the adjacent segment can already be observed.

\section{Acknowledgments:}

We thank Robert Reid Inc. for providing the spinal implants.

\section{References}

\section{Ð⿻: External Link}

1. Abumi K, Panjabi MM, Kramer KM: Biomechanical evaluation of lumbar spinal stability after graded facetectomies. Spine 15:1142-1147, 1990 Pubmed

2. Brodke DS, Dick JC, Kunz DN: Posterior lumbar interbody fusion. A biomechanical comparison, including a new threaded cage. Spine 22:26-31, 1997 Pubmed

3. Buttermann GB, Kahmann RD, Lewis JL: An experimental method for measuring force on the spinal facet joint: description and application of the method. J Biomech Eng 113:374-386, 1991 
4. Chow D, Luk K, Evans J: Effect of short anterior lumbar interbody fusion on biomechanics of neighboring unfused segments. Spine 5:549-555, 1996

5. Cohen JD, Cunningham BW, Shimamoto N: The effect of PLIF cages on biomechanical stability and pedicle screw strain. An in-vitro human cadaveric model. Proceedings of the North American Spine Society 15th Annual meeting, 2000, P125- 127

6. Cunningham BW, Kanayama M, Parker LM: Osteogenic protein versus autologous interbody arthrodesis in the sheep thoracic spine. A comparative endoscopic study using the Bagby and Kuslich interbody fusion device. Spine 24:509-518, 1999 P Pubmed

7. Cunningham BW, Kotani Y, McNulty PS: The effect of spinal destabilization and instrumentation on lumbar intradiscal pressure: an in vitro biomechanical analysis. Spine 22:2655-2663, 1997 口 Pubmed

8. Dekutoski MB, Schendel MJ , Ogilvie JW: Comparison of in vivo and in vitro adjacent segment motion after lumbar fusion. Spine 19:1745-1751, $1994 \square \boxminus$ Pubmed

9. Elias WJ, Simmons NE, Kaptain GJ: Complications of posterior lumbar interbody fusion when using a titanium threaded cage device. J Neurosurg (Spine 1) 93:45-52, 2000

\section{Abstract Full Text PDF}

10. Frymoyer JW, Hanley EN, Howe J: A comparison of radiographic findings in fusion and nonfusion patients ten or more years following lumber disc surgery. Spine 4:435-440, 1979 Ш Pubmed

11. Goel VK, Goyal S, Clark C: Kinematics of the whole lumbar spine. Effect of discectomy. Spine 10:543-554, 1985 G曰 Pubmed

12. Gurr K, McAfee P, Shih C-M: Biomechanical analysis of posterior instrumentation systems after decompressive laminectomy. An unstable calf-spine model. J Bone Joint Surg Am 70:680-691, 1988 밤umed 
13. Heth J A, Hitchon PW, Goel VK: A biomechanical comparison between anterior and transverse interbody fusion cages. Spine 26:E261-E267, $2001 \square \square$ Pubmed

14. Hitchon PW, Goel VK, Rogge TN: In vitro biomechanical analysis of three anterior thoracolumbar implants. J Neurosurg (Spine 2) 93:252-258, 2000

Abstract Full Text PDF

15. Kawakami M, Tamaki T, Ando M: Lumbar sagittal balance influences the clinical outcome after decompression and posterolateral spinal fusion for degenerative lumbar spondylolisthesis. Spine 27:59-64, 2002 口曰 Pubmed

16. Kumar MN, Baklanov A, Chopin D: Correlation between sagittal plane changes and adjacent segment degeneration following lumbar spine fusion. Eur Spine J 10:314-319, 2001 Đubmed

17. Lazennec J-Y, Ramare S, Arafati N: Sagittal alignment in lumbosacral fusion: relations between radiological parameters and pain. Eur Spine J 9:47-55, 2000 1 Pubmed

18. Lee CK: Accelerated degeneration of the segment adjacent to a lumbar fusion. Spine 13:375-377, 1988 ШШ Pubmed

19. Lee CK, Langrana NA: Lumbosacral spinal fusion: A biomechanical study. Spine 9:574-581, 1984 밤

20. Lehmann TR, Spratt KF, Tozzi JE: Long-term follow-up of lower lumbar fusion patients. Spine 12:97-104, 1987 曰曰 Pubmed

21. McAfee PC, Weiland DJ, Carlow JJ: Survivorship analysis of pedicle spinal instrumentation. Spine 16:S422-S427, 1991 ॠ Pubmed

22. Nagata $H$, Schendel MJ, Transfeldt EE: The effects of immobilization of long segments of the spine on the adjacent and distal facet force and lumbosacral motion. Spine 18:2471-2479, 1993 口 Pubmed

23. Oda I, Abumi K, Lu D: Biomechanical role of the posterior elements, 
costovertebral joints, and rib cage in the stability of the thoracic spine. Spine 21:1423-1429, 1996 口ubmed

24. Oda I, Cunningham BW, Buckley R: Does spinal kyphotic deformity influence the biomechanical characteristics of the adjacent motion segments? An in vivo animal model. Spine 24:2139-2146, 1999

25. Okuyama K, Abe E, Suzuki T: Posterior lumbar interbody fusion: a retrospective study of complications after facet joint excision and pedicle screw fixation in 148 cases. Acta Orthop Scand 70: 329-334, 1999 맘 pubmed

26. Panjabi MM: Biomechanical evaluation of spinal fixation devices: I. A conceptual framework. Spine 13: 1129-1134, 1988 Ð Pubmed

27. Rahm MD, Hall BB: Adjacent-segment degeneration after lumbar fusion with instrumentation: a retrospective study. J Spinal Disord 5: 392-400, 1996

28. Schlegel JD, Smith JA, Schleusener RL: Lumbar motion segment pathology adjacent to thoracolumbar, lumbar, and lumbosacral fusions. Spine 21:970-981, $1996 \square$ Pubmed

29. Shono Y, Kaneda K, Abumi K: Stability of posterior spinal instrumentation and its effects on adjacent motion segments in the lumbosacral spine. Spine 23: 1550-1558, $1998 \square$ Pubmed

30. Suk SI, Lee CK, Kim WJ: Adding posterior lumbar interbody fusion to pedicle screw fixation and posterolateral fusion after decompression in spondylolytic spondylolisthesis. Spine 22:210-219, $1997 \square$ Pubmed

31. Umehara S, Zindrick MR, Patwardhan AG: The biomechanical effect of postoperative hypolordosis in instrumented lumbar fusion on instrumented and adjacent spinal segments. Spine 25:1617-1624, $2000 \square$ Pubmed

32. Weinhoffer SL, Guyer RD, Herbert M: Intradiscal pressure measurements above an instrumented fusion. A cadaveric study. Spine 20:526-531, $1995 \square \square$ Pubmed 
33. Yahiro MA: Comprehensive literature review. Pedicle screw fixation devices. Spine 19:S2274-S2278, 1994

34. Yoganandan N, Pintar F, Maiman DJ : Kinematics of the lumbar spine following pedicle screw plate fixation. Spine 18:504-512, 1993 말 Pubmed

Manuscript received November 25, 2002.

Accepted in final form May 27, 2003. 
Figure 1

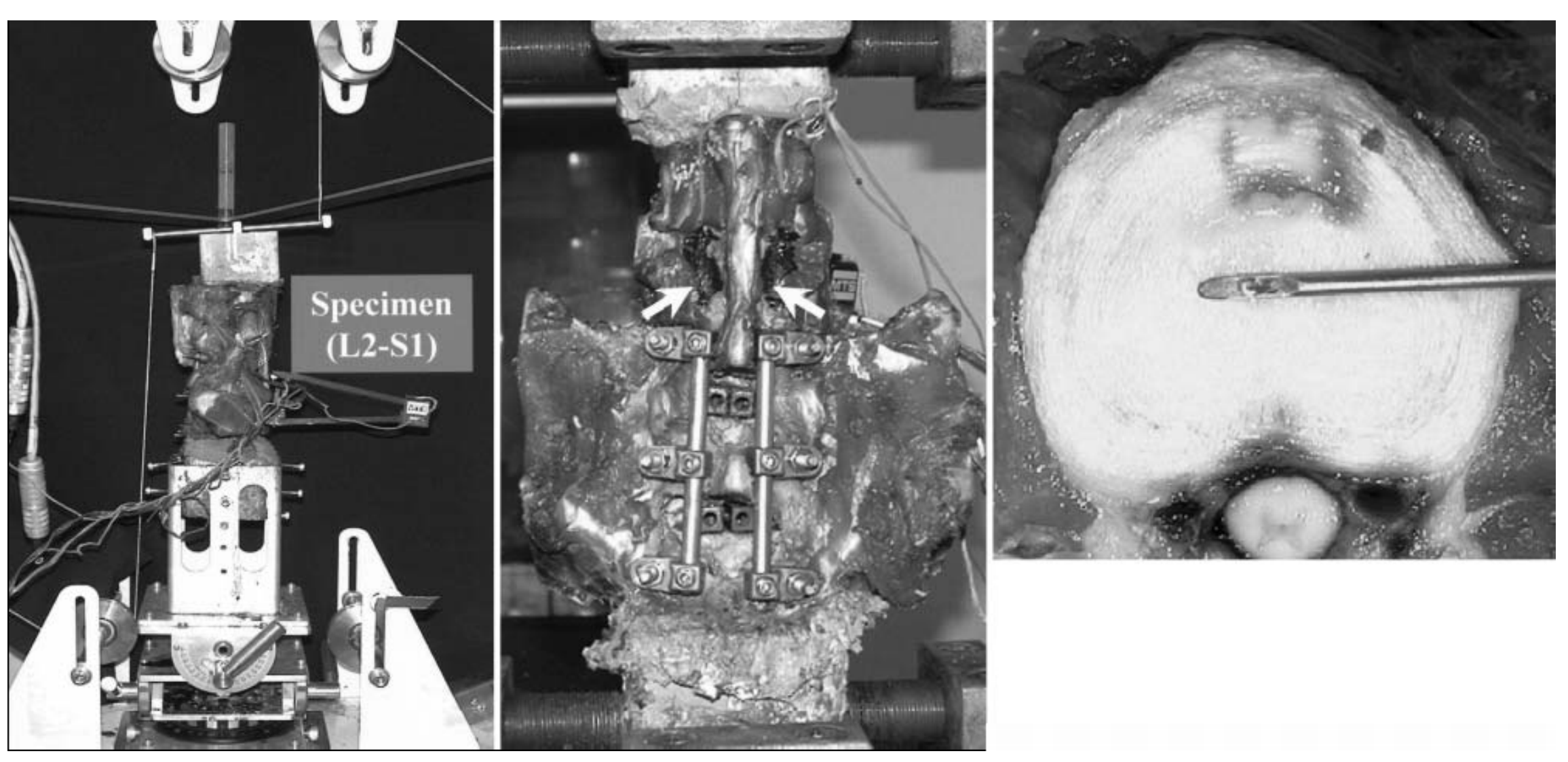


Figure 2

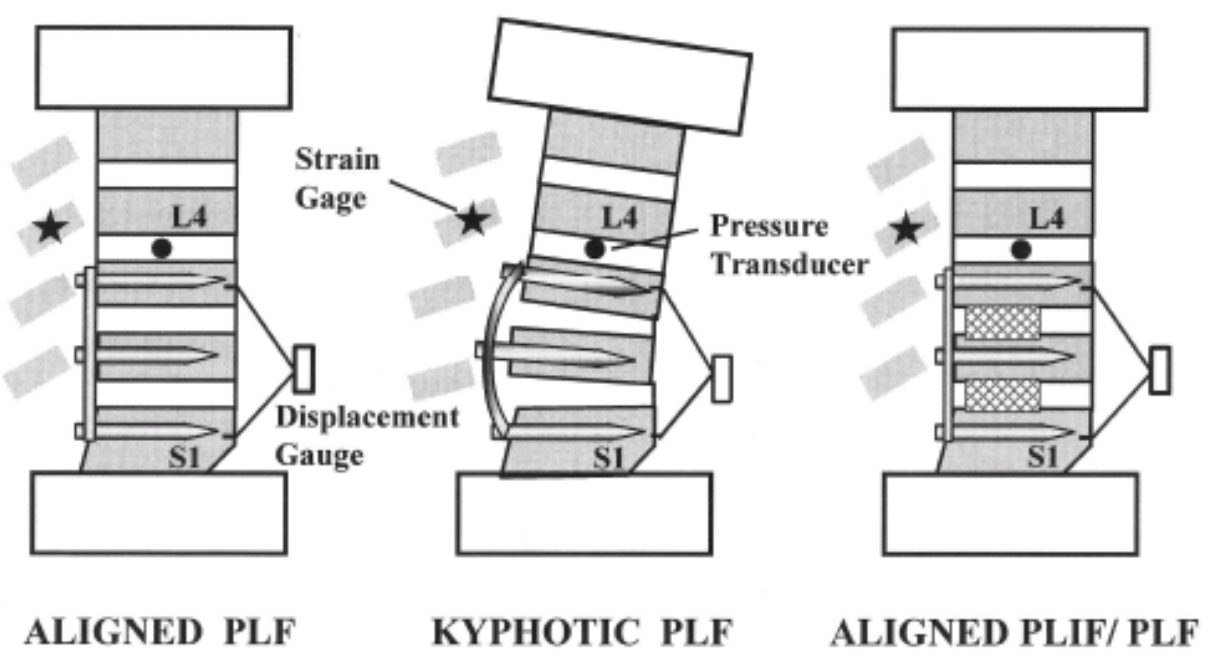

Figure 3
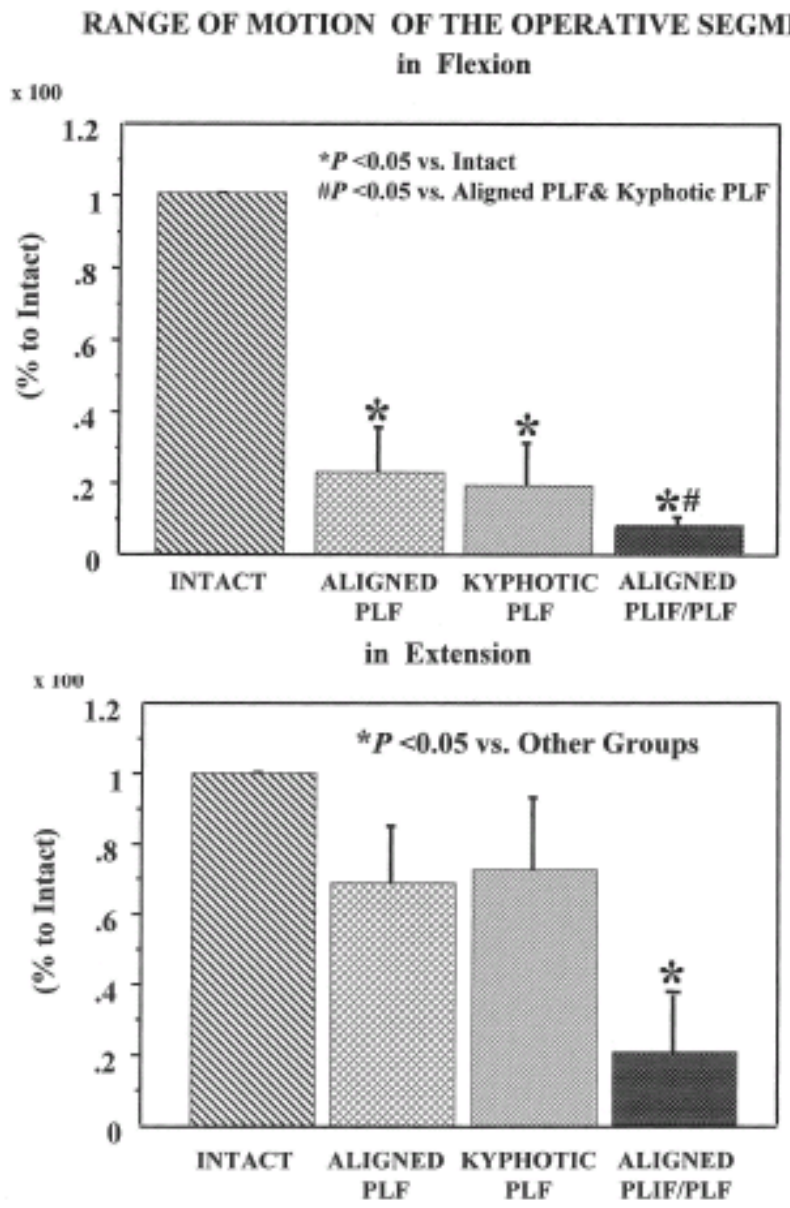
Figure 4

INTRADISCAL PRESSURE in Flexion
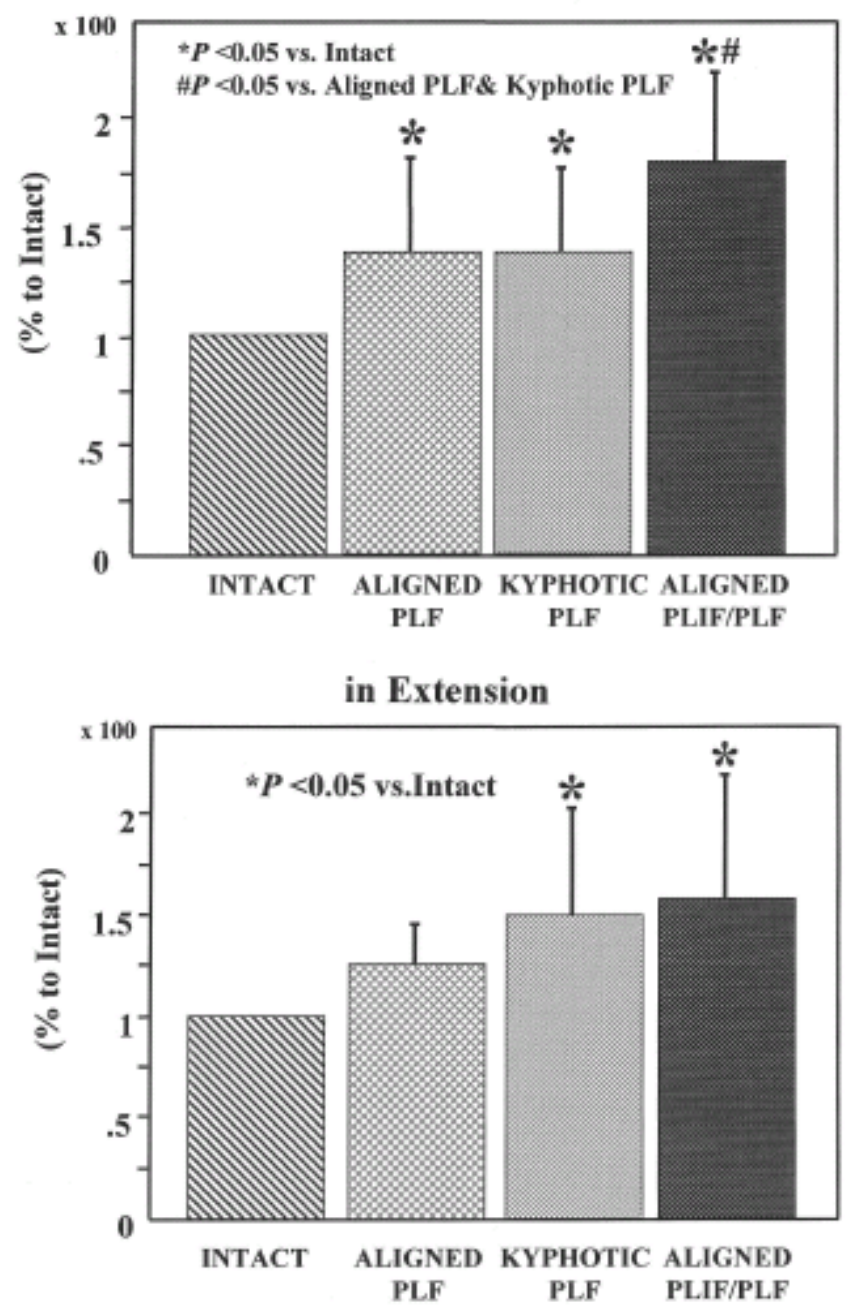
Figure 5
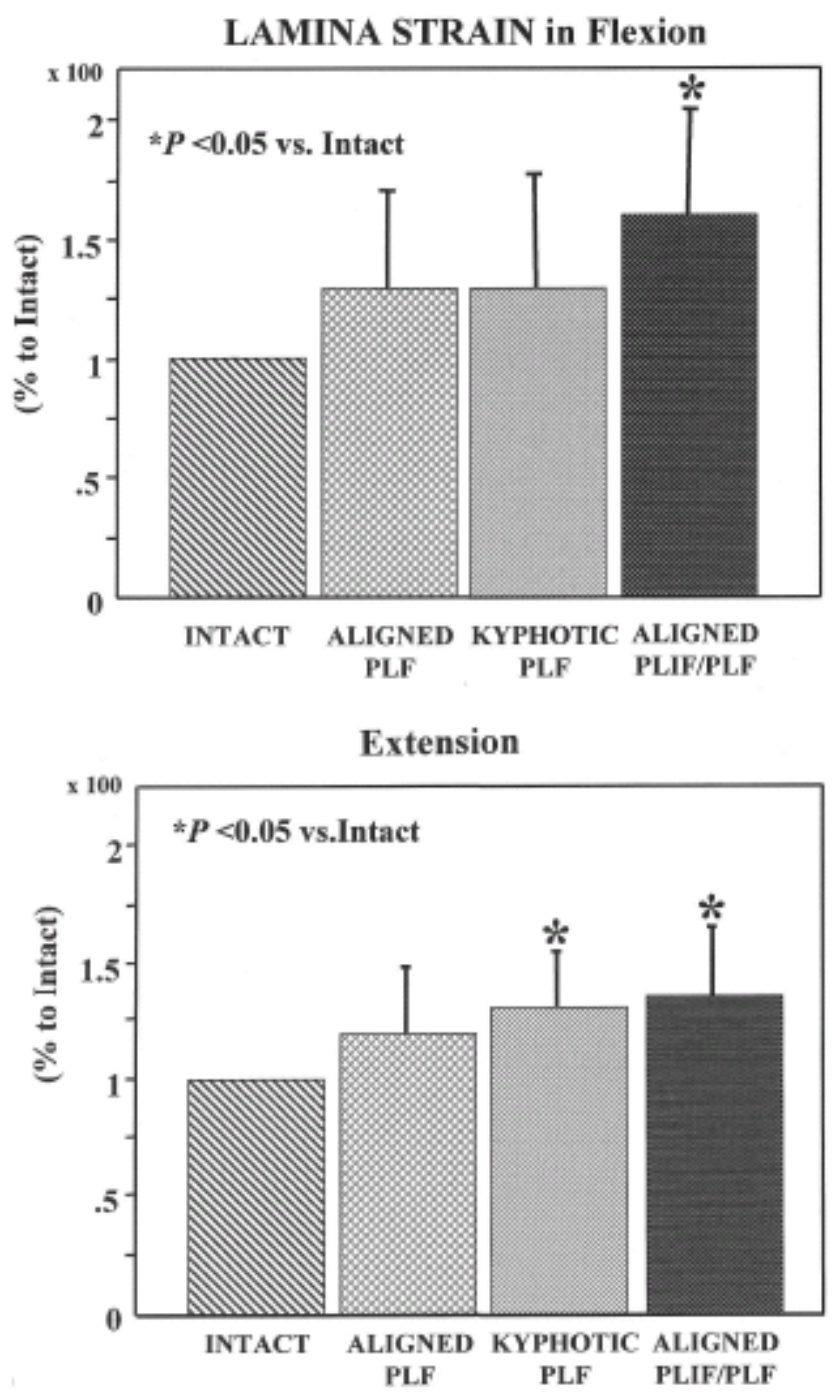